Bull. Korean Math. Soc. 45 (2008), No. 4, pp. 607-614

\title{
COINCIDENCE POINTS OF WEAKLY COMPATIBLE MAPPINGS
}

\author{
YISHENG SONG
}

\begin{abstract}
We present coincidence points and common fixed point results for $(f, g)$-contractive mapping and $(f, g)$-nonexpansive mappings. Our results generalize and complement various known results existing in the literature.
\end{abstract}

\section{Introduction and preliminaries}

Let $K$ be a nonempty subset of a metric space $E$ and $f, g$, and $T$ three selfmaps of $K$, and $C(f, g, T)$ the set of coincidence points of $f, g$, and $T$ (i.e., $C(f, g, T)=\{x \in K ; f x=T x=g x\})$, and $F(T)$ the set of fixed points of $T, F(T)=\{x \in K ; x=T x\}$. We shall denote the closure of $K$ by $\bar{K}$, the boundary of $K$ by $\partial K$ and all positive integer by $\mathbb{N}$. When $\left\{x_{n}\right\}$ is a sequence in $E$, then $x_{n} \rightarrow x$ (respectively, $x_{n} \rightarrow x$ ) will denote strong (respectively, weak) convergence of the sequence $\left\{x_{n}\right\}$ to $x$.

The set $K$ is called (1) q-starshaped with $q \in K$ if $k x+(1-k) q \in K$ for all $x \in K$ and all $k \in[0,1] ;(2)$ convex if $k x+(1-k) y \in K$ for all $x, y \in K$ and all $k \in[0,1]$.

The selfmap $f$ on $K$ is called (3) affine if $K$ is convex and $f(k x+(1-k) y)=$ $k f x+(1-k) f y$ for all $x, y \in K$ and all $k \in[0,1]$; (4) q-affine if $K$ is $q$-starshaped and $f(k x+(1-k) q)=k f x+(1-k) q$ for all $x \in K$ and all $k \in[0,1]$. Note that $f q=q$ whenever $f$ is a $q$-affine selfmap of a $q$-starshaped set $K[1]$.

(5) The selfmap $T$ on $K$ is called $(f, g)$-contraction if, there exists $0 \leq k<1$ such that $d(T x, T y) \leq k d(f x, g y)$ for any $x, y \in K$. If $k=1$, then $T$ is called $(f, g)$-nonexpansive. If $f=g$, then $T$ is called $f$-contraction (or $f$ nonexpansive). If $f=g=I$, an identity operator, then $T$ is called contraction (or nonexpansive).

A mapping $T: K \rightarrow K$ is called (6) continuous if for all $\left\{x_{n}\right\}$ such that $\left\{x_{n}\right\}$ converges to $x$ implies that $\left\{T x_{n}\right\}$ converges strongly to $T x ;$ strongly continuous if for all $\left\{x_{n}\right\}$ such that $\left\{x_{n}\right\}$ converges weakly to $x$ implies that

Received October 26, 2006.

2000 Mathematics Subject Classification. 41A50, 47H10, 54H25.

Key words and phrases. coincidence points, invariant approximations, $(f, g)$-nonexpansive mappings, weakly compatible mappings, common fixed points.

This work is supported by the Chinese National Tianyuan Foundation(10726073). 
$\left\{T x_{n}\right\}$ converges strongly to $T x$; weakly continuous if for all $\left\{x_{n}\right\}$ such that $\left\{x_{n}\right\}$ converges weakly to $x$ implies that $\left\{T x_{n}\right\}$ converges weakly to $T x$. Clearly the strong continuity of $T$ implies both continuity and weakly continuity of $T$ but not conversely [11]; (7) demiclosed at 0 if for every sequence $\left\{x_{n}\right\} \subset K$ such that $\left\{x_{n}\right\}$ converges weakly to $x$ and $\left\{T x_{n}\right\}$ converges strongly to 0 , then $T x=0$.

The map pair $(T, f)$ is called (8) commuting if $T f x=f T x$ for all $x \in K$; (9) $R$-weakly commuting [6] if for all $x \in K$ there exists $R>0$ such that $d(f T x, T f x) \leq R d(f x, T x)$. If $R=1$, then the map pair are called weakly commuting; (10) compatible [2] if $\lim _{n \rightarrow \infty} d\left(T f x_{n}, f T x_{n}\right)=0$ whenever $\left\{x_{n}\right\}$ is a sequence such that $\lim _{n \rightarrow \infty} T x_{n}=\lim _{n \rightarrow \infty} f x_{n}=t$ for some $t$ in $K$; (11) weakly compatible [1] if they commute at their coincidence points, i.e., $f T x=T f x$ whenever $f x=T x$. Suppose that $E$ is compact metric space and both $T$ and $f$ are continuous, then $(f, T)$ compatible equivalent to $(f, T)$ weakly compatible [2, Theorem 2.2, Corollary 2.3].

Suppose that $K$ is $q$-starshaped with $q \in F(f)$ and is both $T$-and $f$-invariant. Then $(T, f)$ are called (12) R-subweakly commuting on $K$ (see $[1,8,9]$ ) if for all $x \in K$, there exists a real number $R>0$ such that $d(f T x, T f x) \leq$ $R \delta(f x,[T x, q])$, where $[T x, q]=\{k T x+(1-k) q ; x \in K, k \in(0,1]\}$ and $\delta(p, K)=\inf _{z \in K} d(z, p)$ for $p \in E$; (13) R-subcommuting [10] on $K$ if for all $x \in K$, there exists a real number $R>0$ such that $d(f T x, T f x) \leq$ $\frac{R}{k} d(k T x+(1-k) q, f x)$ for all $k \in(0,1] ;$ (14) $C_{q}$-commuting [1] if $f T x=$ $T f x$ for all $x \in C_{q}(f, T)$, where $C_{q}(f, T)=\bigcup\left\{C\left(f, T_{k}\right) ; 0 \leq k \leq 1\right\}$ and $T_{k} x=(1-k) q+k T x$. Clearly, $C_{q}$-commuting maps are weakly compatible but not conversely in general. $R$-subcommuting and $R$-subweakly commuting maps are $C_{q}$-commuting but the converse does not hold in general [1]. (15) A normed space $E$ is said to be satisfy Opial's condition if for any sequence $\left\{x_{n}\right\}$ in $E, x_{n} \rightarrow x(n \rightarrow \infty)$ implies

$$
\limsup _{n \rightarrow \infty}\left\|x_{n}-x\right\|<\limsup _{n \rightarrow \infty}\left\|x_{n}-y\right\|, \forall y \in E \text { with } x \neq y .
$$

Hilbert space and the space $l_{p}(1 \leq p<\infty)$ satisfy Opials condition.

During the last decades, the hybrid mapping results have been obtained by many mathematicians (for example, see reference $[1,2,3,4,6,7,8,9,10]$ ).

The aim of this paper is to prove results extending the above mentioned invariant approximation results. In particular, we establish general common fixed point theorems for $(f, g)$-nonexpansive weakly compatible mappings. Our results, on the one hand, extend and unify the work of Al-Thagafi and Shahzad [1], Jungck [3], and on the other hand, provide generalizations and complementarities of the recent work of Jungck and Sessa [4] and Shahzad [7, 8, 9, 10].

\section{Coincidence and common fixed point theorems}

Theorem 2.1. Let $K$ be a subset of a metric space $(E, d)$, and $T, f, g: K \rightarrow K$ be three mappings and $T$ be $a(f, g)$-contraction with a constant $k \in(0,1)$ which 
satisfies $\overline{T(K)} \subset f(K) \bigcap g(K)$. Suppose that either $\overline{T(K)}$ or $f(K)$ or $g(K)$ is complete, then

(i) there exist $z, u, v \in K$ such that $f u=T u=z=T v=g v$, that is, $u \in C(T, f)$ and $v \in C(T, g)$;

If, in addition, $(T, f)$ and $(T, g)$ are weakly compatible, then

(ii) $F(T) \cap F(f) \cap F(g)$ is singleton.

Proof. Take $x_{0} \in K$. As $\overline{T(K)} \subset f(K) \bigcap g(K)$, we can choose a sequence $\left\{x_{n}\right\}$ in $K$ such that $T x_{2 n}=f x_{2 n+1}$ and $T x_{2 n+1}=g x_{2 n+2}$ for all $n \geq 0$. It follows that

$$
d\left(T x_{2 n+1}, T x_{2 n}\right) \leq k d\left(f x_{2 n+1}, g x_{2 n}\right)=k d\left(T x_{2 n}, T x_{2 n-1}\right) .
$$

Similarly, we also have that

$$
d\left(T x_{2 n-1}, T x_{2 n}\right) \leq k d\left(f x_{2 n-1}, g x_{2 n}\right)=k d\left(T x_{2 n-2}, T x_{2 n-1}\right) .
$$

Therefore, for all $n \geq 0$,

$$
d\left(T x_{n+1}, T x_{n}\right) \leq k d\left(T x_{n-1}, T x_{n}\right) \leq k^{n} d\left(T x_{1}, T x_{0}\right) .
$$

Thus,

$$
d\left(T x_{n+p}, T x_{n}\right) \leq \sum_{i=0}^{p} d\left(T x_{n+i}, T x_{n+i+1}\right) \leq \sum_{i=0}^{p} k^{n+i} d\left(T x_{1}, T x_{0}\right) .
$$

Hence, $\left\{T x_{n}\right\}$ is a Cauchy sequence. By the definition of $\left\{T x_{n}\right\}$, then the sequence $\left\{f x_{2 n+1}\right\}$ and $\left\{g x_{2 n+2}\right\}$ are also Cauchy sequences.

Since either $\overline{T(K)}$ or $f(K)$ or $g(K)$ is complete, suppose $f(K)$ is complete. Then $f x_{2 n+1} \rightarrow z \in K$, and by the definition of $\left\{T x_{n}\right\}$, we obtain that

$$
g x_{2 n}, f x_{2 n+1}, T x_{n} \rightarrow z \in \overline{T(K)} \subset f(K) \bigcap g(K) .
$$

Hence there exist $u, v \in K$ such that $f u=z=g v$. Then as $n \rightarrow \infty$,

$$
d\left(T x_{2 n+1} T v\right) \leq k d\left(f x_{2 n+1}, g v\right)=k d\left(f x_{2 n+1}, z\right) \rightarrow 0 .
$$

Thus $T x_{n} \rightarrow T v=z=g v$. Similarly, we also can show that $T u=z=f u$. (i) is proved.

Finally we prove (ii). As $(T, f)$ and $(T, g)$ are weakly compatible and $g v=$ $T v=z=T u=f u$, then

$$
g z=g T v=T g v=T z=T f u=f T u=f z .
$$

We claim that $z$ is a common fixed point of $T, f, g$. Since

$$
d(z, T z)=d(T u, T z) \leq k d(f u, g z)=k d(z, T z),
$$

then $z=T z$, i.e., $z \in F(T) \cap F(f) \cap F(g)$. If there exists another point $v \in K$ such that $v=T v=g v=f v$, then

$$
d(z, v)=d(T z, T v) \leq k d(f z, g v)=k d(z, v) .
$$

Hence $z=v$. The proof is complete. 
Theorem 2.1 contains the Banach Contraction Principle as a special case $(f=g=I)$. It generalizes Al-Thagafi and Shahzad [1, Theorem 2.1 ]. It also extends Shahzad [8, Lemma 2.1] and Pant [6, Theorem 1].

Theorem 2.2. Let $K$ be a nonempty $q$-starshaped subset of a normed space $E$, and $T, f, g: K \rightarrow K$ three mappings. Assumed that $T$ is a $(f, g)$-nonexpansive mapping, and $f$ and $g$ are $q$-affine and $\overline{T(K)} \subset f(K) \cap g(K)$. Suppose that either $\overline{T(K)}$ or $f(K)$ or $g(K)$ is compact, then

(i) there exist $z, u, v \in K$ such that $f u=T u=z=T v=g v$;

If, in addition, $(T, f)$ and $(T, g)$ are weakly compatible and $f f x=f x$ for all $x \in C(T, f)$, then

(ii) $F(T) \cap F(f) \cap F(g) \neq \emptyset$.

Proof. Choose a sequence $\left\{k_{n}\right\} \subset(0,1)$ such that $\lim _{n \rightarrow \infty} k_{n}=1$. For each $n$, define $T_{n}$ by

$$
T_{n} x=\left(1-k_{n}\right) q+k_{n} T x, \forall x \in K .
$$

Then, for each $n, \overline{T_{n}(K)} \subset f(K) \cap g(K)$ by $q$-starshapedness of $K$ and $q$-affiness of $f$ and $g$. And also for all $x, y \in K$,

$$
\left\|T_{n} x-T_{n} y\right\|=k_{n}\|T x-T y\| \leq k_{n}\|f x-g y\|,
$$

so $T_{n}$ is $(f, g)$-contractive mapping with contractive coefficient $k_{n} \in(0,1)$. As either $\overline{T(K)}$ or $f(K)$ or $g(K)$ is compact, then $\overline{T(K)}$ or $f(K)$ or $g(K)$ is complete [11]. It follows from Theorem 2.1(i) that for each $n$, there exist $x_{m(n)}, x_{t(n)} \in K$ such that

(2.1) $f x_{m(n)}=k_{n} T x_{m(n)}+\left(1-k_{n}\right) q=y_{n}=g x_{t(n)}=k_{n} T x_{t(n)}+\left(1-k_{n}\right) q$.

It follows from the compactness of either $\overline{T(K)}$ or $f(K)$ or $g(K)$ that there exist $\left\{y_{n_{i}}\right\} \subset\left\{y_{n}\right\}$ and $z \in K$ such that

$$
\begin{gathered}
y_{n_{i}}=f x_{m\left(n_{i}\right)}=g x_{t\left(n_{i}\right)} \rightarrow z(i \rightarrow \infty), \\
T x_{m\left(n_{i}\right)}=T x_{t\left(n_{i}\right)}=\frac{y_{n_{i}}-\left(1-k_{n_{i}}\right) q}{k_{n_{i}}} \rightarrow z \in \overline{T(K)} .
\end{gathered}
$$

And also $z \in f(K) \cap g(K)$ by $\overline{T(K)} \subset f(K) \cap g(K)$. Hence there exist $u, v \in K$ such that $z=f u=g v$. As $i \rightarrow \infty$,

$$
\left\|T u-T x_{t\left(n_{i}\right)}\right\| \leq\left\|f u-g x_{t\left(n_{i}\right)}\right\|=\left\|z-g x_{t\left(n_{i}\right)}\right\| \rightarrow 0,
$$

therefore, $T x_{t\left(n_{i}\right)} \rightarrow T u=z$, i.e., $z=T u=f u$. Similarly, we also can show that $T v=z=g v$. (i) is proved.

Subsequently, we show (ii). It follows from (i) that there exist $z, u, v \in K$ such that $f u=T u=z=g v=T v$. Since $(T, f)$ and $(T, g)$ are weakly compatible and $f f x=f x$ for all $x \in C(T, f)$, then

$$
f z=f T u=T f u=T z=T g v=g T v=g z
$$

and

$$
f z=f f u=f u=z .
$$


Thus $z=f z=g z=T z$, which proves (ii).

Theorem 2.3. Let $K$ be a nonempty $q$-starshaped subset of a Banach space $E$, and $T, f, g: K \rightarrow K$ three mappings. Assumed that $T$ is a $(f, g)$-nonexpansive mapping, and $f$ and $g$ are $q$-affine and $\overline{T(K)} \subset f(K) \cap g(K)$. Suppose that one of the following conditions is satisfied:

(a) $T$ is strongly continuous and $K$ is weakly compact;

(b) $f$ or $g$ is strongly continuous and $K$ is weakly compact;

(c) $\overline{T(K)}$ is weakly compact and E satisfies Opial's condition.

Then (i) $C(T, f, g) \neq \emptyset$;

If, in addition, $(T, f)$ and $(T, g)$ are weakly compatible and $f f x=f x$ for all $x \in C(T, f)$, then

(ii) $F(T) \cap F(f) \cap F(g) \neq \emptyset$.

Proof. Let $\left\{k_{n}\right\}$ and $\left\{T_{n}\right\}$ be defined as in Theorem 2.2. Then a similar argument shows that there exist $x_{m(n)}, x_{t(n)} \in K$ such that

$$
f x_{m(n)}=k_{n} T x_{m(n)}+\left(1-k_{n}\right) q=y_{n}=g x_{t(n)}=k_{n} T x_{t(n)}+\left(1-k_{n}\right) q .
$$

Suppose the condition (a) holds. Since $\left\{x_{m(n)}\right\} \subset K$ together with the weak compactness of $K$, then there exists $y \in K$ and $\left\{x_{m\left(n_{i}\right)}\right\} \subset\left\{x_{m(n)}\right\}$ such that $x_{m\left(n_{i}\right)} \rightarrow y(i \rightarrow \infty)$. It follows from the strong continuity of $T$ that

$$
T x_{m\left(n_{i}\right)} \rightarrow T y \in \overline{T(K)} \subset f(K) \cap g(K) .
$$

Thus there exist $u, v \in K$ such that $T y=f u=g v$, and noticing $k_{n} \rightarrow 1$,

$$
f x_{m\left(n_{i}\right)}=g x_{t\left(n_{i}\right)}=y_{n_{i}}=k_{n_{i}} T x_{m\left(n_{i}\right)}+\left(1-k_{n_{i}}\right) q \rightarrow T y .
$$

We claim that $T u=T y=f u$. Indeed, since as $i \rightarrow \infty$

$$
\left\|T u-T x_{t\left(n_{i}\right)}\right\| \leq\left\|f u-g x_{t\left(n_{i}\right)}\right\|=\left\|T y-g x_{t\left(n_{i}\right)}\right\| \rightarrow 0,
$$

then $T x_{t\left(n_{i}\right)} \rightarrow T u=T y$. Similarly, we also can show that $T v=T y=g v$. (i) is proved.

Suppose the condition (b) holds. Assumed that $f$ is strongly continuous, then $g x_{t\left(n_{i}\right)}=f x_{m\left(n_{i}\right)} \rightarrow f y$. Since as $i \rightarrow \infty$

$$
\left\|T y-T x_{t\left(n_{i}\right)}\right\| \leq\left\|f y-g x_{t\left(n_{i}\right)}\right\|=\left\|f y-f x_{m\left(n_{i}\right)}\right\| \rightarrow 0
$$

then $T x_{t\left(n_{i}\right)} \rightarrow T y$, that is, $T$ is strongly continuous at $y$. It follows from (a) that we also reach our objective.

Suppose the condition (c) holds. By the weak compactness of $\overline{T(K)}$, there exists $y \in K$ and $\left\{T x_{m\left(n_{i}\right)}\right\} \subset\left\{T x_{m}(n)\right\}$ such that $T x_{m\left(n_{i}\right)} \rightarrow z(i \rightarrow \infty)$. Therefore by $k_{n} \rightarrow 1$, we have

$$
f x_{m\left(n_{i}\right)}=g x_{t\left(n_{i}\right)}=k_{n_{i}} T x_{m\left(n_{i}\right)}+\left(1-k_{n_{i}}\right) q \rightarrow z .
$$

Since weak closedness of subset of $E$ implies closedness in Banach space $E$ $[11,5]$, then $z \in \overline{T(K)} \subset f(K) \cap g(K)$. Thus $\exists u, v \in K$ such that $z=f u=g v$. As $\left\{T x_{n}\right\}$ is bounded by the weak compactness of $\overline{T(K)}$, then

$$
\left\|f x_{m}(n)-T x_{m}(n)\right\|=\left(1-k_{n}\right)\left\|T x_{m}(n)-q\right\| \rightarrow 0(n \rightarrow \infty) .
$$


We claim that $T v=z$. Suppose not, By $E$ satisfying Opial's condition, we get

$$
\begin{aligned}
\limsup _{i \rightarrow \infty}\left\|T x_{m\left(n_{i}\right)}-z\right\| & <\limsup _{i \rightarrow \infty}\left\|T x_{m\left(n_{i}\right)}-T v\right\| \\
& \leq \limsup _{i \rightarrow \infty}\left\|f x_{m\left(n_{i}\right)}-g v\right\|=\limsup _{i \rightarrow \infty}\left\|f x_{m\left(n_{i}\right)}-z\right\| \\
& \leq \limsup _{i \rightarrow \infty}\left\|f x_{m\left(n_{i}\right)}-T x_{m\left(n_{i}\right)}\right\|+\limsup _{i \rightarrow \infty}\left\|T x_{m\left(n_{i}\right)}-z\right\| \\
& =\limsup _{i \rightarrow \infty}\left\|T x_{m\left(n_{i}\right)}-z\right\| .
\end{aligned}
$$

Which is a contradiction. Hence, $z=T v=g v$. Similarly, we also can show that $z=T u=f u$. (i) is proved.

It follows from the similar argumentation of Theorem 2.3(ii) that $T u \in$ $F(T) \cap F(f) \cap F(g)$. Which finishes the proof.

Theorem 2.4. Let $K$ be a nonempty q-starshaped subset of a normed space $E$, and $T, f, g: K \rightarrow K$ three mappings which satisfy that $T$ is a $(f, g)$ nonexpansive mapping and $\overline{T(K)} \subset f(K) \cap g(K)$. Suppose that $(T, f)$ and $(T, g)$ are $C_{q}$-commuting, and $f$ and $g$ are $q$-affine, and one of the three mappings $T, f, g$ is continuous. If either $\overline{T(K)}$ or $f(K)$ or $g(K)$ is compact, then $F(T) \cap F(f) \cap F(g) \neq \emptyset$.

Proof. Let $\left\{k_{n}\right\} \subset(0,1)$ such that $\lim _{n \rightarrow \infty} k_{n}=1$. For each $n$, define $T_{n}$ by

$$
T_{n} x=\left(1-k_{n}\right) q+k_{n} T x, \forall x \in K .
$$

It follows from the similar argumentation of Theorem 2.2 that $\overline{T_{n}(K)} \subset f(K) \cap$ $g(K)$ for each $n$ and $T_{n}$ is $(f, g)$-contractive mapping with contractive coefficient $k_{n} \in(0,1)$. Since $(T, f)$ and $(T, g)$ are $C_{q}$-commuting, and $f$ and $g$ are $q$-affine, then $q \in F(f) \cap F(g)[1]$, and furthermore, for all $T_{n} x=f x=g x$, we have

$T_{n} f x=\left(1-k_{n}\right) q+k_{n} T f x=\left(1-k_{n}\right) f q+k_{n} f T x=f\left(\left(1-k_{n}\right) q+k_{n} T x\right)=f T_{n} x$.

Namely, $\left(T_{n}, f\right)$ is weakly compatible. Similarly, $\left(T_{n}, g\right)$ is weakly compatible also. It follows from Theorem 2.1(ii) that for each $n$, there exists unique $x_{n} \in K$ such that

$$
x_{n}=f x_{n}=g x_{n}=k_{n} T x_{n}+\left(1-k_{n}\right) q .
$$

The alike argument as Theorem 2.2(i) implies that there exist $z, u, v \in K$ and $\left\{x_{n_{i}}\right\} \subset\left\{x_{n}\right\}$ such that $T u=f u=z=T v=g v$, and $x_{n_{i}}=f x_{n_{i}}=g x_{n_{i}} \rightarrow z$ and $T x_{n_{i}} \rightarrow z$ as $i \rightarrow \infty$. As $C_{q}$-commuting of $(T, f)$ and $(T, g)$ implies weakly compatible [1], then

$$
f z=f T u=T f u=T z=T g v=g T v=g z .
$$

It follows from continuity of either $T$ or $f$ or $g$ that either $T x_{n_{i}} \rightarrow T z$ or $f x_{n_{i}} \rightarrow f z$ or $g x_{n_{i}} \rightarrow g z$. Hence

$$
z=T z=f z=g z .
$$

This completes the proof. 
Corollary 2.5. Let $K$ be a nonempty q-starshaped subset of a normed space $E$, and $T: K \rightarrow K$ a nonexpansive mapping and $\overline{T(K)} \subset K$. If $\overline{T(K)}$ is a compact subset of $E$, then $F(T) \neq \emptyset$.

Theorem 2.6. Let $K$ be a nonempty q-starshaped subset of a Banach space $E$, and $T, f, g: K \rightarrow K$ three mappings which satisfy that $T$ is a $(f, g)$ nonexpansive mapping and $\overline{T(K)} \subset f(K) \cap g(K)$. Suppose that $(T, f)$ and $(T, g)$ are $C_{q}$-commuting and $f$ and $g$ are $q$-affine, and $T$ is strongly continuous, and either $K$ or $\overline{T(K)}$ or $f(K)$ or $g(K)$ is weakly compact. Then $F(T) \cap F(f) \cap F(g) \neq \emptyset$.

Proof. Let $\left\{k_{n}\right\}$ and $\left\{T_{n}\right\}$ be defined as in Theorem 2.4. Then a similar argument shows that for each $n$, there exists unique $x_{n} \in K$ such that

$$
x_{n}=f x_{n}=g x_{n}=k_{n} T x_{n}+\left(1-k_{n}\right) q .
$$

The alike argument as Theorem 2.3(i) implies that there exist $z, u, v \in K$ and $\left\{x_{n_{i}}\right\} \subset\left\{x_{n}\right\}$ such that $T u=f u=z=T v=g v$, and $x_{n_{i}}=f x_{n_{i}}=g x_{n_{i}} \rightarrow z$

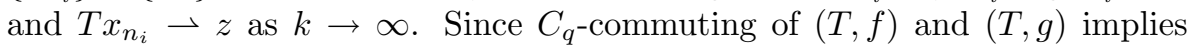
weakly compatible [1], then

$$
f z=f T u=T f u=T z=T g v=g T v=g z .
$$

As $T$ is strongly continuous together with $x_{n_{i}} \rightarrow z$, then $T x_{n_{i}} \rightarrow T z$. By $T x_{n_{i}} \rightarrow z$, we have $z=T z=f z=g z$. The proof is completed.

Remark. 1. Both Theorem 2.2 and Theorem 2.4 generalize Al-Thagafi and Shahzad [1, Theorem 2.2], Jungck [3, Theorem 3.1] and Shahzad [8, Lemma 2.2].

2. Both Theorem 2.3 and Theorem 2.6 extend and improve Al-Thagafi and Shahzad [1, Theorem 2.4] and Shahzad [7, Theorem 3].

Acknowledgments. The author would like to thank editors and the anonymous referee for their valuable suggestions which helps to improve this manuscript.

\section{References}

[1] M. A. Al-Thagafi and N. Shahzad, Noncommuting selfmaps and invariant approximations, Nonlinear Anal. 64 (2006), no. 12, 2778-2786.

[2] G. Jungck, Common fixed points for commuting and compatible maps on compacta, Proc. Amer. Math. Soc. 103 (1988), no. 3, 977-983.

[3] __ Coincidence and fixed points for compatible and relatively nonexpansive maps, Internat. J. Math. Math. Sci. 16 (1993), no. 1, 95-100.

[4] G. Jungck and S. Sessa, Fixed point theorems in best approximation theory, Math. Japon. 42 (1995), no. 2, 249-252.

[5] R. E. Megginson, An Introduction to Banach Space Theory, Graduate Texts in Mathematics, 183. Springer-Verlag, New York, 1998.

[6] R. P. Pant, Common fixed points of noncommuting mappings, J. Math. Anal. Appl. 188 (1994), no. 2, 436-440.

[7] N. Shahzad, On R-subcommuting maps and best approximations in Banach spaces, Tamkang J. Math. 32 (2001), no. 1, 51-53. 
[8] , Invariant approximations and R-subweakly commuting maps, J. Math. Anal. Appl. 257 (2001), no. 1, 39-45.

[9] _ Invariant approximations, generalized l-contractions, and $R$-subweakly commuting maps, Fixed Point Theory Appl. (2005), no. 1, 79-86.

[10] $\ldots$ Noncommuting maps and best approximations, Rad. Mat. 10 (2000/01), no. 1, 77-83.

[11] S. P. Singh, B. Watson, and P. Srivastava, Fixed Point Theory and Best Approximation: The KKM-map Principle, Kluwer Academic Publishers, Dordrecht, 1997.

College of Mathematics and Information Science

HENAN NORMAL UNIVERSITY

453007, P. R. CHINA

E-mail address: songyisheng123@yahoo.com.cn 